\title{
Modeling and Evaluation of Single Event Effects in 90nm MOS Devices
}

\author{
Zhenhuan $\mathrm{Wu}$, Yuhe $\mathrm{Li}^{+}$, Zhendong Qiao, Jiahua Kou, Pengnian Yang \\ Department of Precision Instrument, Collaborative Innovation Center of Advanced Nuclear Energy \\ Technology, Tsinghua University, Beijing, China
}

\begin{abstract}
With the simulation results of the single event effects (SEE) of 90nm metal-oxide-semiconductor (MOS), the influence of incident angle of leakage current was studied. Based on the noise theory of carrier number fluctuation and mobility fluctuation, a semi-empirical formula with respect to both the pre-irradiation $1 / \mathrm{f}$ noise and the sensitivity of single event effect was derived in details. In 90nm MOS drain in single event effects there exist a current pulse peak, with which the critical value of $1 / \mathrm{f}$ noise amplitude was proposed. By comparison between the threshold value and pre-irradiation 1/f noise, a non-destructive method was proposed to preliminary predict and estimate the single event effect for $90 \mathrm{~nm}$ MOS devices. The experiments are carried out and the results agree well with the established formula.
\end{abstract}

Keywords: $90 \mathrm{~nm}$ MOS, single event effect, $1 /$ f noise.

\section{Introduction}

Single event effects are caused by a single high-energy particle from the space and particle strike causes direct ionization effect or nuclear reactions to generate and cumulate electric charges. When it comes to that the sensitive regions of the semiconductor devices deposit sufficient electric charges, soft errors including memory bit flip and transient may occur. [1] At the nanoscale, single event effects can lead to critical issues in space environments. For example, with their own sizes becoming smaller and smaller, devices themselves may no longer be able to attenuate the current pulses induced by single event effects. As a consequence, even very small charge can change the work status of the devices in space environments.

According to the noise theory, the characterization and experiment evaluation of the irradiation damage is an effective and non-destructive method. Recent studies show that the low-frequency noise plays an indispensable role in practical applications since it becomes an important limitation of the device performances. [2.3] 1/f noise can reflect the internal defects and the internal characteristics of small changes in semiconductor. It has been found that the amplitude of pre-irradiation $1 / \mathrm{f}$ noise is closely related to electrical parameter drift. However, most of the published studies on radiation so far, involved with $1 / \mathrm{f}$ noise have mainly focused on the proportional relationship between $1 / \mathrm{f}$ noise amplitude and threshold voltage drift [4-8]. In some other previous papers, mathematical statistics method has been used to evaluate the radiation resistance of device. [9] Single event effects are regarded as a special case in radiation effects, and the charge collection is the fundamental cause. The primary failure mechanism is the transient current. The damage is non-destructive, which can be repaired by rewriting the affected unit. [10] Nonetheless, the above method does not have been applied to evaluate the single event effects at the nanoscale. Moreover, by the new technology the feature size can be more narrowed and the radiation effects will also have different

\footnotetext{
+ Corresponding author. Tel.: + 8610-62794630; fax: +8610-62784691

E-mail address: liyuhe@mail.tsinghua.edu.cn
} 
influences at the nanoscale. [1,11,12] In conclusion, present studies have not established the precise quantitative relationship between pre-irradiation $1 / \mathrm{f}$ noise and single event effects.

Based on the simulation of single event effects of $90 \mathrm{~nm}$ MOS, the phenomenon and mechanism has been studied .Using the mathematic model of pre-irradiation $1 / \mathrm{f}$ noise on the prediction of single event effect in 90 nm MOS device.

\section{Simulation}

TCAD (Technology Computer Aided Design) is a kind of software technology related to semiconductor process and device simulation. With the development of computer technology, it is a new research direction that single event effects of devices at the nanoscale can be simulated by TCAD software. Computer aided software simulation can make up for deficiency in radiation resistance designs and single event effects and predict the ground simulation experiments. 3D simulation can truly reflect the working status of the devices at the nanoscale. [13] In this paper, we use the TCAD software of silvaco company to simulate single event effects on devices at the nanoscale.

A 3D $90 \mathrm{~nm}$ MOS model in Figure 1 has been built for simulation. Main model parameters are the following: Gate oxide thickness, $5 \times 10^{-9} \mathrm{~m}$. Deep, $1.8 \times 10^{-8} \mathrm{~m}$, Interface doping concentration, $10^{17} \mathrm{~cm}^{-3}$, Substrate doping, $6 \times 10^{16} \mathrm{~cm}^{-3}$.

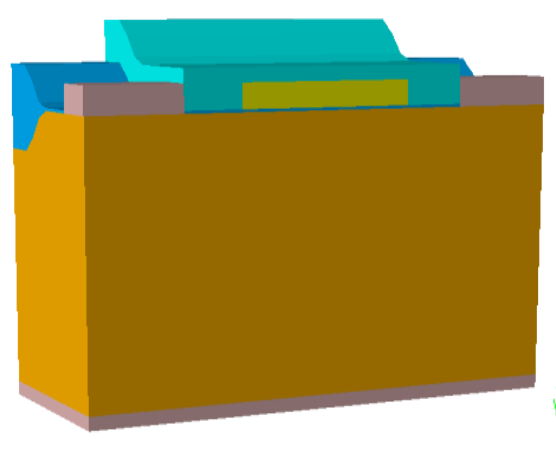

(a)

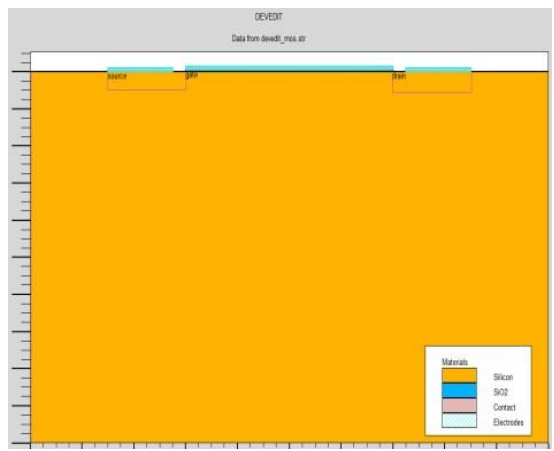

(b)

Fig. 1: 90nm MOS simulation (a) 3D (b) $2 \mathrm{D}$

Based on different linear energy transfers (LET), incident angles, incident locations of particles can be simulated and single event effects can be studied on $90 \mathrm{~nm}$ MOS. This paper mainly studies that semiconductor devices response to the particle energy deposition under different conditions such as MOS drain current transient cure. Simulation steps are the following: defining the particle LET, incident points, exit points and irradiation time, then selecting an appropriate numerical method, and run the program. Figure 2 shows the particle trajectory on $90 \mathrm{~nm}$ MOS, Figure 2 shows the influence curve of the device collecting drain current under different incident angles and positions. The red curve respects the normal incident current and the green curve respects the oblique incident current.

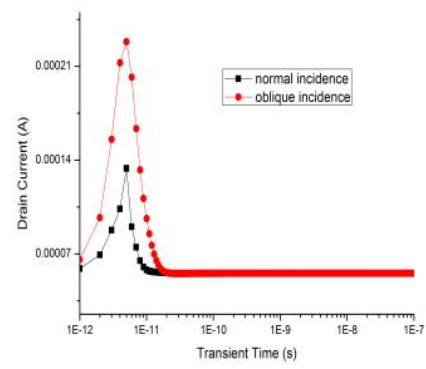

(a)

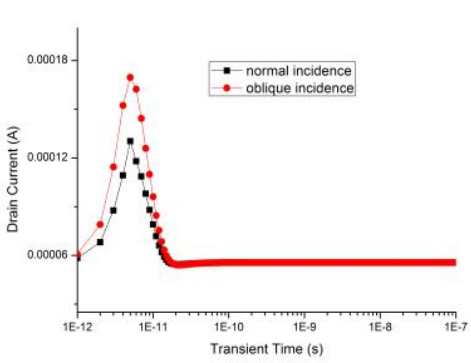

(b)

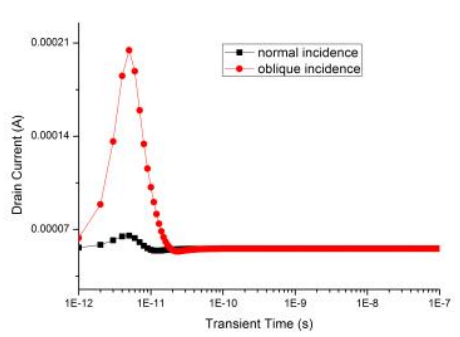

(c)

Fig. 2: the influence drain current curve under different incident angle and position. (a) Drain (b) Gate (c) Source

Incident angles affect drain current in the single particle incident process. From Figure 2 we can see, no matter where particles enter from, the transient response peak generated by the oblique incidence is higher than a normal incidence. For example, the oblique incident current peak of which particles enter from the drain is $2.28 \times 10^{-4} \mathrm{~A}$, and the normal incident current peak is $1.34 \times 10^{-4} \mathrm{~A}$. Namely, the ratio is $1.70: 1$. The 
smallest ratio is only 1:30:1 when particles enter from the gate. The largest gap ratio from the source of incidence is 3.10:1. The particles pass through a longer path when the incidence is oblique, which produce more hole-electron pairs that start to drift under the electric field by the direct ionization effect or nuclear reactions. As a result, the sensitive area accumulates more charge and produces larger current, and finally the transient current peak is higher.

Table 1: The ratio of the oblique and normal incidence

\begin{tabular}{cccc}
\hline Position & drain & gate & source \\
\hline Peak current & $1.77: 1$ & $1.30: 1$ & $3.10: 1$ \\
Path length & $1.57: 1$ & $1.10: 1$ & $1.57: 1$ \\
\hline
\end{tabular}

Date analysis provides the ratio of the path lengths of the normal and oblique incidence is $1.57: 1$ and the ratio, which is similar to the ratio of peak current, becomes 1.10:1 when particles enter from the drain and gate. The path length of source incidence is the same as the drain incidence, but the ratio is 3.5:1 and much higher than drain incidence. Besides, there are other influential factors considering the influence of the path. In theory, the drain is sensitive to the node of MOS devices, the sensitive node produces drain current when drain incidence, eventually makes logic state change or damage. In fact, MOS devices are upset when the source is incident, and the reason is that source creates a large number of carriers when the particle energy is large enough to produce hole-electron pairs. According to the funnel effect principle, the areas of dense carrier is similar to the conductor, and therefore, when the carrier has sufficient density, the drain is capable of absorbing the ionization of carriers and produces drain current. The absorption process of the carrier is accelerated under the funnel effect, and if the carrier is struck more, the avalanche multiplication effect will occur. As a result, the oblique incident current presents a multiplication phenomenon. The normal incident carrier has less capacity to trigger the funnel effect, therefore its drain is very weak.

\section{Method}

All of the recent studies about $1 / \mathrm{f}$ noise are based on the fluctuation of carrier numbers and the mobility fluctuation mechanism. Parts of the studies consider separately in the two mechanisms of which is in a dominant position. In this paper, we claim that the $1 / \mathrm{f}$ noise caused by the joint action by number fluctuation and mobility fluctuation mechanism [14]. Oxide trapping and launching the channel of the carrier cause carrier number fluctuation, which causes channel mobility fluctuation by coulomb scattering, which leads to channel current fluctuation.

According to the carrier numbers and mobility fluctuation theories, oxide trap unit area causes current fluctuation:

$$
\frac{\delta I_{d}}{I_{d}}=\left\lfloor\frac{1}{\Delta N} \cdot \frac{\delta \Delta N}{\delta \Delta N_{t}}+\frac{1}{\mu_{e f f}} \cdot \frac{\delta \mu_{e f f}}{\delta \Delta N_{t}}\right\rfloor \delta \Delta N_{t}
$$

Channel current: $I_{d}=W \mu q N E_{x} . W$ is the channel width, $\mu$ is the channel mobility, $N$ is the unit area of the channel number of carrier, $N={ }^{k_{B} T C_{i}} / q, \Delta N=N W \Delta x, \Delta N_{t}=N_{t} W \Delta x, N_{t}$ is the unit area occupied on the number of trap.

According to Matthiessen law, mobility can be expressed as followed:

$$
\frac{1}{\mu_{\text {eff }}}=\frac{1}{\mu_{n}}+\alpha N_{t}
$$

According to the noise power spectrum method and compared with the normal form of the $1 / \mathrm{f}$ noise power spectrum: $S_{V}(x, f)=\frac{B}{f^{\gamma}}$, the noise amplitude $B$ and frequency index $\gamma$ can be obtained by following formula: ${ }^{[7,8]}$

$$
\begin{aligned}
B=\frac{2 q k^{2} T^{2} I_{d} \mu_{e f f}}{\alpha L^{2} \tau_{0}^{\frac{\eta}{\theta}} \omega^{\frac{\eta}{\theta}+1}} & \frac{\pi}{\eta \cos \left(\frac{\eta \pi}{2 \theta}\right)} \int_{0}^{V_{d}} N_{t}\left(E_{f n}\right)\left(1+\alpha \mu_{e f f} N R^{-1}\right)^{2} \frac{R^{2}}{N} d V \\
\gamma & =\frac{\eta}{\boldsymbol{\theta}}+1
\end{aligned}
$$


$\mathrm{R}$ is the proportional constant of carrier number and the number of occupied trap is $R=\frac{\delta \Delta N}{\delta \Delta N_{t}}$. k is the Boltzmann constant, $\mathrm{T}$ is the indoor temperature.

$\left.N_{t}\left(E_{f n}\right)=N_{0} \exp \left[\xi\left(E_{F}-E_{i}\right)\right] 1-\exp \left(-\frac{\Delta E_{\max }}{k_{B} T} \frac{\eta}{\theta}\right)\right], E_{f n}$ is the Electron quasi-Fermi level. In the practical engineering application, Noise parameters are extracted by measuring the source voltage noise of MOS devices and using the appropriate method.

In order to accurately and quantitatively evaluate the single event effects on MOS devices, we claim a threshold value $B_{0}$ of $1 / \mathrm{f}$ noise amplitude, and construct the following criteria: pre-irradiation $1 / \mathrm{f}$ noise amplitude exceeds the threshold value. Considering the condition that the device has a sensitive single event effect, poor ability to resist radiation and reliability cannot reach the requirements.

Based on the simulated results particles entering from any angels and positions, current produces transient pulse, and the peak value can be greatly changed at least more than 2 times. Therefore, it is assumed that when the device current suddenly increases to more than 2 times, that is, the possibility of single particle effects is low and the performance of resist radiation is weak. According to the evaluation criteria, the leakage current fluctuation is greater than C. C is a proportional constant of drain current change value and original current value, of which the value is usually take 2, expressed as follow:

$$
\frac{\delta I_{d}}{I_{d}}>C
$$

$B_{0}$ can be derived from the formula (1)(3)(5):

$$
B_{0}=\frac{2 q k^{2} T^{2} I_{d} \mu_{e f f}}{\alpha L^{2} \tau_{0}^{\frac{\eta}{\theta}} \omega^{\frac{\eta}{\theta}+1}} \frac{\pi}{\eta \cos \left(\frac{\eta \pi}{2 \boldsymbol{\theta}}\right)} N_{t}\left(E_{f n}\right) \frac{C^{2}}{N} V_{d}
$$

\section{Experimental Results}

Experimental samples are prepared by three groups of $90 \mathrm{~nm}$ standard process $\mathrm{N}$ channel enhanced MOS, the ratio of channel width and length is $W / L=250: 90$, and preliminary test is verified in the heavy ion accelerator. Flux is $2000 \mathrm{~s} / \mathrm{cm}^{2}$, LET is $31 \mathrm{Mev} \cdot \mathrm{cm}^{2} / \mathrm{g}$, the cumulative number of measurement is $1.001 \mathrm{E}+5$, ion range is $110 \mu \mathrm{m}$. Experiments are carried out at the room temperature, and the working state and the logic state will change after radiation.

According to the $1 / \mathrm{f}$ noise measured before the radiation of the $90 \mathrm{~nm}$ device, the frequency index $\gamma$ and noise amplitude $B$ can be obtained by an appropriate method. Parameters $\eta$ and $N_{t}\left(\mathrm{E}_{\mathrm{fn}}\right)$ can be obtained by formula (3) and (4), and eventually plugged into formula (6), and the the critical value can be calculated.

Table 2.Calculation result

\begin{tabular}{cccc}
\hline Devices Number & $\mathbf{1}$ & $\mathbf{2}$ & $\mathbf{3}$ \\
\hline $\boldsymbol{B}$ & $1.20 \times 10^{-11}$ & $4.89 \times 10^{-12}$ & $1.91 \times 10^{-11}$ \\
$\boldsymbol{B}_{0}$ & $2.48 \times 10^{-13}$ & $1.10 \times 10^{-13}$ & $3.95 \times 10^{-13}$ \\
$\Delta \boldsymbol{B}=\boldsymbol{B}-\boldsymbol{B}_{0}$ & $1.1752 \times 10^{-11}$ & $4.78 \times 10^{-12}$ & $1.87 \times 10^{-11}$ \\
Abnormal times & 39 & 29 & 46 \\
\hline
\end{tabular}

According to the experimental results, the pre-irradiation noise amplitude is larger than the value by $1 \sim$ 2 orders of magnitude, and single event effects will occur after radiation. Assumptions consist with expectations, then the difference between the pre-irradiation noise amplitude and critical value is smaller and the number of abnormal times becomes less.

\section{Conclusions}

A kind of evaluating method and simulation of single event effects are introduced. Based on simulation technology, there exists a current pulse peak in $90 \mathrm{~nm}$ MOS drain in single event effects, with which the critical value of $1 / \mathrm{f}$ noise amplitude is proposed. By comparison between the critical value and pre- 
irradiation 1/f noise, a non-destructive method to preliminarily predict and estimate the single event effects for $90 \mathrm{~nm}$ MOS devices is proposed in this paper.

\section{Acknowledge}

This research is supported by the State Key Laboratory of Precision Measurement Technology and Instruments, the National Natural Science Foundation of China (Grant No. 51275259 and No. 61362035) and the Collaborative Innovation Center of Advanced Nuclear Energy Technology.

\section{References}

[1] M. A. Aguirre, V. Baena, J. Tombs, M. Violant. A New Approach to Estimate the Effect of Single Event Transients in Complex Circuits. IEEE Trans . On Nucl. Sci, 2007, 54(3):1018-1024.

[2] D. M. Fleetwood. Evidence that similar point defects cause 1/f noise and radiation-induced-hole trapping in MOS transistors. Phys. Rev. Lett. 1990, 64 (5): 579-582.

[3] H. D. Xiong, D. M Fleetwood, J. R. Schwark. Low-frequency noise and radiation response of buried oxides in SOI nMOS transistors dern Differential Geometry. Noise in Devices and Circuits. 2004, 151(2):118-124.

[4] D. M. Fleetwood, et al. Effect of radiation-induced interface traps on 1/f noise in MOSFETs. IEEE Trans . On Nucl. Sci, 1992, 39(12):2178-2185.

[5] M. R. Shaneyfelt, J. R Schwank, D. M. Fleetwood, P. S. Winokur. Effects of irradiation temperature on MOS radiation response. IEEE Trans. On Nucl. Sci, 1998, 45(3):1372-1378.

[6] J. A. Babcockt. Effects of ionizing radiation on the noise properties of DMOS power transistors. IEEE Trans .On Nucl. Sci, 1992, 39(12):2012-2017

[7] S. Q. Peng, L. Du, Y.Q. Zhuang. J.L. Bao, W.H. Chen. Radiation degradation model of metal-oxidesemiconductor field effect transistor based on pre-irradiation 1/f noise. Phy. Sin. 2008, 57(8):5205-5211.

[8] Z. G. Zhao. M. NDE of power MOSFET's resisting radiation ability. Ph .D. Dissertation (Xian. P.R), 2008

[9] J. L. Bao. Reliability Diagnostic Methods by Means of Noise in Semiconductor Devices. Ph.D. Dissertation (Xian .P.R ), 2005.

[10] H. Andrew, A. Len (translated by W. Guan ). Handbook of radiation effects. BICE press, 2000.

[11] D.E. Fullkerson, Member. An Engineering Model for Single-Event-Effects and Soft Error Rates in Bulk CMOS IEEE Trans . On Nucl. Sci, 2011, 58(2):506-515.

[12] S. D. Gupta, A. F.Witulski, B. L. Bhuva, et al. Effect of well and substrate potential modulation on single event pulse shape in deep submicron CMOS. Phys. Rev. IEEE Trans. On Nucl. Sci, 2007, 54(6):2407-2412.

[13] S. Q. Chen. Ph.D. Dissertation (Chinese Academy of Sciences ), 2010.

[14] M. H. Tsai. et al, Effect of radiation-induced interface traps on 1/f noise in MOSFETs. IEEE Trans . On Nucl. Sci, 1992, 39(12):2178-2185. 